\title{
Variations
}

Variations

Revue internationale de théorie critique

18 | 2013

"La poésie est une arme chargée de futur »

\section{Entretien avec Sergio Zamora, écrivain chilien, à propos de son livre Sept heures entre les mains de la Dina}

\section{Hélène Chatroussat et Sergio Zamora}

\section{OpenEdition}

Journals

Édition électronique

URL : http://journals.openedition.org/variations/655

DOI : $10.4000 /$ variations. 655

ISSN : 1968-3960

Éditeur

Les amis de Variations

Référence électronique

Hélène Chatroussat et Sergio Zamora, «Entretien avec Sergio Zamora, écrivain chilien, à propos de son livre Sept heures entre les mains de la Dina », Variations [En ligne], 18 | 2013, mis en ligne le 31 mai 2013, consulté le 03 mai 2019. URL : http://journals.openedition.org/variations/655 ; DOI : 10.4000/ variations.655

Ce document a été généré automatiquement le 3 mai 2019.

Les ami•e•s de Variations 


\title{
Entretien avec Sergio Zamora, écrivain chilien, à propos de son livre Sept heures entre les mains de la Dina
}

\author{
Hélène Chatroussat et Sergio Zamora
}

\section{RÉFÉRENCE}

Sergio Zamora, Sept heures entre les mains de la Dina, éditions Florent Massot, 1993, rééd. Yvelinedition, 2010

Sergio Zamora, Après Septembre, chronique de la résistance, éditions Florent Massot, 1995

\section{NOTE DE L'ÉDITEUR}

Au moment du sanglant coup d'Etat militaire du 11 septembre 1973, Sergio Zamora avait 24 ans et militait au Parti socialiste chilien, le parti du président Salvador Allende. Celuici avait été élu démocratiquement trois ans plus tôt et trouvera la mort le jour même du coup de force de la junte. Les partis de gauche ne s'étaient pas du tout préparés à cette éventualité d'un coup d'Etat. Il avait été concocté minutieusement à la fois par des secteurs de l'armée et par des experts des Etats-Unis. L'initiative des putschistes fut soutenue activement par le patronat, la droite et l'extrême-droite ainsi que par la hiérarchie de l'Eglise catholique. La terreur déferla sur le pays. Des milliers de militants et sympathisants de gauche furent assassinés et torturés au cours des dix-sept années qu'allait durer la dictature militaire dirigée par Pinochet.

Tandis que son parti, tout comme les autres partis de gauche, était désarticulé par la répression et que de nombreux dirigeants et militants se réfugiaient dans les ambassades et prenaient le chemin de l'exil, Sergio Zamora fit le choix, avec d'autres militants du PS, 
du MIR, du PC ou du MAPU, de rester au Chili. En dépit de son inexpérience de la vie clandestine, il décida de résister et de dénoncer sur place les crimes et les mensonges de la dictature. Une vie d'angoisse commença alors pour lui, pour ses proches, ses amis, ses camarades, une vie émaillée de drames, de trahisons et aussi d'actes de courage et de solidarité magnifiques. Il tiendra et agira pendant vingt mois et quatre jours avant d'être arrêté le 15 mai 1975 par des hommes de la Dina (Direction nationale d'Intelligence), la «Gestapo » chilienne placée directement sous les ordres de Pinochet.

Sergio Zamora a écrit un récit détaillé, lucide et particulièrement émouvant de cette période de résistance personnelle et collective dans Après Septembre, chronique de la résistance (éditions Florent Massot, 1995) auquel il fait plusieurs fois référence au cours de l'entretien qui suit.

Après son arrestation, grâce à un concours de circonstances mais aussi à sa sagacité et à sa connaissance des gens à qui il avait affaire, il a réussi, après avoir été torturé pendant plusieurs heures à l'électricité, à tromper la vigilance de ses gardiens et à se réfugier dans un endroit où siégeait un local du Comité pour la Paix lié à l'Eglise. Comme il l'a écrit dans sa présentation de Sept heures entre les mains de la Dina (éditions Florent Massot en 1993, réédition Yvelinedition en 2010) : « En plus de sauver ma vie, mon geste provoqua un durcissement de l'attitude de l'Eglise catholique envers la dictature. En m'évadant le jour même de mon interrogatoire, je constituai la première preuve vivante des atteintes à la dignité humaine commises par l'utilisation de la torture. Les cas de torture connus à l'époque - deux ans après le coup d'Etat - se comptaient par milliers mais restaient difficiles à prouver. Les victimes des nombreux services répressifs, quand elles n'étaient pas ensuite assassinées, étaient gardées au secret le temps nécessaire à la disparition de toute séquelle physique.»

Les hommes d'Eglise mis devant le fait accompli refusèrent de livrer Sergio Zamora à la Dina et obtinrent de Pinochet qu'il soit expulsé avec son épouse et sa fille, soit un mois après son arrestation.

Hélène Chatroussat

Hélène : Sergio, peux-tu nous dire quelques mots sur ce que tu as fait, sur ce que tu as écrit dans ton livre Sept heures entre les mains de la Dina?

Sergio : D'abord, il faut savoir que ce livre raconte mon expérience en tant que jeune responsable socialiste au Chili. J'ai été nommé dans les jours qui ont suivi le coup d'Etat militaire de septembre 1973, par l'ancienne direction de la section du parti de Santiago qui était la plus importante du PS. J'ai été nommé comme l'un des cinq responsables du Parti socialiste dans cette région. Tous les anciens dirigeants sont partis en exil, c'était des gens connus... et nous avons créé une direction clandestine moins nombreuse, moins connue mais qui avait quand même une certaine connaissance des rouages $d u$ parti. Donc cela m'a permis de faire ma tâche.

J'ai été nommé responsable «militaire» au PS, ce qui était une responsabilité complètement... irresponsable parce que nous n'avions aucune capacité dans le domaine militaire. Moi, je l'ai acceptée parce que je me souviens, à l'époque, on avait honte au sujet de ce coup d'Etat pour lequel la gauche avait tellement mal réagi. J'en suis convaincu, je ne sais pas pour les autres camarades; on a énormément parlé de tout cela. Je n'ai jamais été partisan de la lutte armée. Par contre j'étais partisan de lutter le jour du coup d'Etat. Et le fait ne pas l'avoir fait, cela m'a chamboulé dans mes convictions. Si j'ai accepté d'être responsable militaire après le coup d'Etat, c'était dû à cela. Disons qu'on aurait dû le faire - on ne l'a pas fait - pas en tant que personnes car 
moi et d'autres, nous étions décidés à nous battre - mais l'ensemble de la gauche chilienne ne l'a pas fait. Les partis de l'Unité populaire de la gauche chilienne ont décidé de ne pas se battre et le coup d'Etat est arrivé.

Bref, j'ai été nommé responsable de la région de Santiago. Cela a duré presque deux ans et a été interrompu le 15 mai 1975 quand je me suis fait arrêter. Donc là, inutile de dire ce que vous connaissez par le livre, ce jour-là j'ai eu la chance de pouvoir m'échapper et ensuite je suis arrivé en France le 15 juin 1975. À l'époque on avait une activité militante assez forte. Alors il fallait s'organiser à l'intérieur mais aussi à l'extérieur pour pouvoir aider le parti au Chili. Toutes les années de 1975 à 1980, j'ai eu une activité assez forte comme militant chilien en exil, pour envoyer de l'argent, envoyer des gens... j'avais donc toute une activité en France.

J'ai travaillé comme ouvrier chez un marchand de vins. C'était un travail assez difficile. J'ai fait cela pendant deux ans. Ensuite je suis venu à Paris et j'ai travaillé au Centre Pompidou.

Mon activité militante s'est amenuisée à mesure que passait le temps. Cette activité au PS s'est arrêtée l'année 85 parce que la situation au Chili avait changé et moi, je n'y suis pas retourné. Je n'y suis allé qu'en 2000, entre autre parce que ma famille s'opposait à mon retour. Elle savait sûrement que j'aurais été embêté par la police.

Ma famille ici s'est agrandie. Avec mon épouse, nous sommes arrivés en France avec une fille qui avait neuf mois. Ensuite nous avons eu un fils et une fille qui sont nés ici ; donc j'avais une famille, pas nombreuse mais quand même une famille importante dont il fallait s'occuper.

Et je me suis plus ou moins décidé à avoir une activité en tant qu'écrivain dans les années 90. En fait j'ai toujours écrit et j'ai toujours su que j'allais écrire un jour. Mais pour une façon ou une autre, je ne sais pas pourquoi, je ne l'ai pas fait d'une façon systématique ; on pense toujours qu'on a toute la vie devant soi.

Souvent on se dit cela, je le ferai plus tard, jusqu'à ce qu'arrive le moment où l'on se rend compte qu'il faut faire les choses parce que le temps passe trop vite.

Et moi, cela m'est arrivé dans les années 2000 à peu près. Donc dès ce moment-là, je me suis dit, il faut que je le fasse et à partir de 2005 je me suis mis à écrire de façon plus ou moins « professionnelle».

J'ai sorti mon premier livre, Sept heures entre les mains de la Dina en 1993, puis en 1995, Après septembre. Ensuite j'ai eu un long moment d'écriture mais je n'ai rien édité. Le troisième livre est en espagnol, Viaje al pais de la lluvia, le quatrième est de la prose poétique et ensuite j'ai écrit surtout pour des revues littéraires et quelques petites choses par-ci par-là, et mon premier livre de nouvelles à la fin de l'année 2007. J'ai aussi sorti un livre de nouvelles en 2008 et un troisième en 2009.

En 2010 il y a eu la réédition de Sept heures entre les mains de la Dina et j'ai publié Les guerriers de la pluie, Brève histoire des Mapuche (1536-1810) et en 2011 la suite de cette histoire, Les guerriers du crépuscule (1810-1884) ainsi que Cronicas del barrio Recoleta sur un quartier populaire du Chili.

En 2012 je n'ai rien édité car j'ai passé toute l'année à écrire. Profitant que c'était l'anniversaire du coup d'Etat en 2013 , le $40^{\mathrm{e}}$ anniversaire, je dois sortir deux livres, un de nouvelles et un sur le coup d'Etat du 11 septembre. 
$H$ : Passer de l'action militante au récit oral, puis au récit écrit, qu'est-ce que cela change pour toi ?

S : C'est à la fois la même chose et pas la même chose. C'est important cela pour moi. De l'activité militante, je suis entré au Parti socialiste en 1969, et comme j'étais un des seuls à savoir écrire à la machine, à l'époque il n'y avait pas d'ordinateur, je faisais les rapports. Déjà au lycée, je faisais de l'information pour l'activité politique. J'avais mon interprétation dans la tête, j'avais vingt ans, bon, mais je faisais cela. Ensuite une grande partie de mon travail a toujours été d'écrire, de faire des rapports, des synthèses, tout cela...

Après le coup d'Etat, quand j'ai donc été nommé « chef du département militaire », mon activité consistait à faire des pamphlets. J'ai fait cela tout de suite.

$\mathrm{H}$ : En fait, il y avait une différence entre rapports et pamphlets.

$\mathrm{S}$ : Oui, on essayait de faire des feuilles d'une seule page pour combler la lacune dans l'information. Au Chili l'information était aux mains de la dictature. Alors nous faisions cela: on commentait un fait politique pour lequel on donnait une autre version; et fallait que cela tienne dans une seule feuille et que cela corresponde à la réalité. On dénonçait, donc c'était toujours un travail de synthèse.

$H$ : Aviez-vous des réactions sur ces pamphlets?

S: La principale réaction qu'on a eue, c'est celle des militaires! Bien sûr, ils nous cherchaient énormément pour cela. Notre parti était le seul à faire cette activité et on l'a fait pendant deux ans. Quand j'ai été arrêté, cela s'est arrêté.

La première fois, c'était fin décembre 73 , on a jeté des pamphlets en plein centre de Santiago. C'est moi qui les ai jetés depuis une fenêtre et en mai 75 , là il y avait 500 personnes pour le faire, tout le monde aguerri, chaque équipe avait son matériel. C'était un travail assez intéressant. C'était tout à fait dans l'idée de votre association, c'est-àdire donner la parole à ceux qui ne l'ont pas. Et nous, on faisait cela. C'est vrai qu'il y avait quand même un dessein caché. Personnellement, je pensais qu'il fallait préparer les gens militairement. Comme on n'avait pas les armes, on faisait une activité qui était risquée mais on faisait équipe. Si on se faisait arrêter, on pouvait le payer de sa vie. Alors on ne jetait pas des balles mais des mots avec nos feuilles.

$H$ : Les mots sont des armes, Sergio.

$\mathrm{S}$ : Oui, voilà.

$H$ : Qu'est-ce qui t'a poussé à écrire ce récit autobiographique? Est-ce que tu l'as écrit d'abord en espagnol ou tout de suite en français.

S: J'ai un ami qui s'appelait Traful Alvarez. Il est décédé maintenant. C'était un Argentin qui était au MIR, un sociologue, qui a travaillé longtemps avec les Mapuche au Chili. On s'est retrouvés par hasard à Paris. On habitait à $200 \mathrm{~m}$ l'un de l'autre, à côté des Buttes Chaumont. C'était un grand cadre, un intellectuel et aussi un artiste peintre. On se voyait tout le temps.

Un jour il m'a dit quelque chose d'assez intéressant : « Il faut que tu écrives ton histoire parce que c'est une histoire positive, qui se finit bien. Souvent quand on écrit sur la torture, c'est une tragédie, ce sont des choses qui se finissent mal, alors que pour toi c'est différent, tu t'es échappé.» C'était en 1978-79. "Je te conseille de l'écrire maintenant car il peut t'arriver quelque chose par la suite (rires)». 


\section{$H$ : C'était un humoriste...}

$\mathrm{S}$ : Moi, j'avais gardé une copie de la vingtaine de pages que j'avais envoyée à mes camarades au Chili et en France. Et un jour, j'ai commencé à les étoffer en mettant des détails. Mais c'était resté sans lendemain. L'année 1993 qui était le vingtième anniversaire du coup d'Etat, je me suis dit, pourquoi je ne le ferai pas à ce moment-là ? J'ai commencé à l'écrire en espagnol. Je l'ai montré à quelques personnes qui s'y sont intéressées et à des éditeurs. Mais en fait, en France, les gens ne lisent pas l'espagnol, les éditeurs non plus. Donc j'ai repris le travail et je l'ai écrit en français, aidé par mes filles qui sont bilingues et par une amie. Enfin bref, tout le monde s'y est mis.

De ce premier texte, j'ai fait une cinquantaine de copies et les ai envoyées à une cinquantaine d'éditeurs. Il n'y en a qu'un qui m'a répondu, un jeune éditeur. Il s'appelle Florent Massot. C'est comme cela que le livre est sorti. C'était un tirage à 10000 exemplaires et tout s'est vendu. C'est ce qui m'a encouragé à continuer d'écrire. Florent m'a conseillé de demander une bourse au CNL et grâce à cela, j'ai continué d'écrire.

$\mathrm{H}$ : Je me demande comment, dans la mesure où tu étais confronté aux tortionnaires qui voulaient te tuer sans laisser de témoins, tu as tenu bon. Pendant les sept heures où tu es resté au centre de torture Villa Grimaldi, comment as-tu détecté des fissures dans ce dispositif mortel?

$\mathrm{S}$ : Disons que, vous savez, ce ne sont pas des choses...

$H$ : C'est trop dur d'en parler - on arrête. C'est déjà dur à lire ce récit.

$\mathrm{S}$ : Non, pas du tout. Non, c'est que ce sont des choses difficiles à formuler. Je crois déjà que quand on écrit quelque chose, on a déjà digéré une situation, une information, donc on l'élabore et on l'exprime. Ce qui parfois ne répond pas exactement à ce qui est arrivé.

Par exemple, les gens qui ont lu mon livre m'ont souvent dit: «on est étonné par ta capacité à faire face. » Ce qui ne correspond pas du tout à ce qui s'est passé vraiment. Parce que sur le coup, on ne réfléchit pas trop. Moi en tout cas, je ne réfléchissais pas beaucoup. Sur le coup, tout était par intuition en fait. Disons, comment j'ai tenu bon pendant sept heures, c'est parce que je ne pouvais pas faire autrement (rire). Ce ne sont pas des choix. On est poussé.

La question s'est posée une seule fois quand on était dans la voiture, dans la rue. Qu'estce qui va se passer si je sors de la voiture ? J'y ai pensé une seule fois vite fait dans ma tête parce que c'était risqué. Je répondais à une situation en essayant de trouver la solution pour pouvoir me faire tuer. Je voulais me faire tuer.

$\mathrm{H}$ : Cela tu l'écris plusieurs fois mais surtout, tu tenais à la vie.

\section{$\mathrm{S}$ : Oui, c'est vrai.}

$\mathrm{H}$ : Tu avais une colonne sentimentale intuitive exceptionnelle. Tu avais des motivations profondes qui font que tu voulais à la fois mourir et surtout pas mourir.

$\mathrm{S}$ : Oui, tout à fait. À un moment donné, je voulais, parce qu'il y avait le métro, des grands trous, et j'avais pensé y dévier la voiture. Je voulais faire ça. À un moment donné je voulais le faire, je n'ai pas osé. Ce n'est pas parce que je tenais à la vie, c'est parce que je n'ai pas osé, ce n'est pas pareil.

Par la suite j'ai pensé à cela, que je tenais à la vie, je crois que non. Je devais passer à l'acte. Ce n'est pas évident. Je savais qu'il fallait faire quelque chose pour ne pas entrer à la Villa. Je ne l'ai pas fait à ce moment, je n'étais peut-être pas prêt, c'est possible. 
Pourquoi je ne l'ai pas fait? Parce que j'avais la trouille. Mais avoir la trouille à ce moment-là, c'était une façon de tenir à la vie (rire), donc ce n'est pas évident.

$H$ : Tout au long du cauchemar, tu te parles à toi-même. C'est dur à lire et le lecteur doit se reprendre. Sans cesse, tu captes des informations qui sont loin de toi, les paroles des tortionnaires, les bruits, les odeurs, tu t'en sers.

S : Oui.

H: J'ai trouvé que le noyau dur, c'était ce que tu te disais à toi-même. Cela te permettait peut-être de faire le bon choix et non l'irrationnel.

$S$ : Je ne sais pas si cela se trouve dans la version en espagnol ou si j'ai réécrit cela par la suite en français. J'ai lu quelque part que quand on a très peur, on voit brusquement tout défiler lentement dans notre tête. Cela m'était arrivé quelques fois. Quand j'étais étudiant au Chili et dans les bagarres qui étaient parfois assez violentes, je voyais les choses défiler très lentement. Cela me permettait d'agir en conséquence et de prendre la bonne décision. Je me suis toujours demandé si c'était une chose que j'imaginais et en parlant avec d'autres personnes, elles m'ont dit que non. À un moment donné, le cerveau est capable de passer les choses au ralenti. Je crois que ce que j'ai vécu là j'avais tellement peur - que j'ai eu cette possibilité.

$\mathrm{H}$ : et tellement mal...

$\mathrm{S}$ : Pas tellement mal mais je voyais les choses plus lentement et cela me permettait de prendre mon temps pour répondre. Je crois qu'au moment où on m'a arrêté, je me suis vu de haut, j'ai vu la scène comme si j'étais au-dessus ; après cela s'est passé. J'ai vu des choses bizarres. Ce sont des choses bizarres qui arrivent dans la tête d'une personne quand elle a peur.

$\mathrm{H}$ : Le suspense de vie et de mort a duré un mois. C'est seulement le 15 juin que ton épouse, ton bébé et toi, vous avez été saufs mais en exil. Une fois réfugié au local du Comité Pro Paz, tu te trouves sous la protection de plusieurs églises. Cependant tu restes sur tes gardes.

S: Quand je me suis échappé dans le local des Comités pour la Paix, il y avait des gens qui n'étaient pas très clairs. Ce n'est pas une impression. Ce sont des gens qui travaillaient là qui me l'ont dit : « Fais attention, avec certains, on ne sait pas trop... ». Mais j'ai passé dix jours avec des religieuses américaines. C'était des personnes excellentes. L'une d'elles s'est fait assassiner des années après au Salvador par les escadrons de la mort du major D'Aubuisson.

Il y a beaucoup de gens qui m'ont aidé. Je me souviens, tout au début, il y avait beaucoup de monde à cet endroit, donc il y avait des gens pas clairs. Disons en général, j'étais sur mes gardes parce qu'il fallait que je le sois ! Je n'ai pas eu tort.

Quand on m'a emmené dans un local qui était un hospice, j'étais avec des gens qui allaient mourir du cancer. Pourquoi on m'a emmené là-bas? Parce que le médecin du cardinal Silva Henriquez, du nom de Luchini et qui était un homme de droite, voulait me voir mais pas dans un lieu caché. Il voulait me voir dans un hôpital ou quelque chose qui y ressemble. Donc ils m'ont emmené dans ce lieu pour les mourants. Ce qui a provoqué des qui pro quo parce que j'étais visité par des religieux - alors que je suis athée - les malades ont cru que j'étais mourant. Par la suite, quand j'ai commencé à marcher, les gens disaient, « c'est un miracle ! ».

$H$ : Quelque part, ils ne croyaient pas si bien dire (rires). Je voudrais revenir sur les tortionnaires à qui tu as eu affaire, la manière dont tu t'es adressé à eux a dû jouer dans le 
fait que tu les as trompés finalement. Qu'est-ce que tu as perçu, car on fait rapidement une erreur d'évaluation dans une situation pareille?

$\mathrm{S}$ : Oui, la première chose qui a joué c'est que j'ai vécu dans des quartiers populaires. J'ai participé à énormément de bagarres, encore plus quand j'étais jeune. Dans les bagarres de rue, qu'est-ce qu'on apprend ? On apprend que « quand faut y aller, faut y aller », il ne faut pas faire semblant, sinon on se fait casser la gueule, alors on y va à fond.

La deuxième chose qui m'a beaucoup aidé, c'est le livre de Gilles Perrault, L'Orchestre rouge. Je l'ai lu tout de suite après le coup d'Etat. Cela circulait sous le manteau et cela m'avait marqué. Je me suis inspiré de cela. C'est-à-dire qu'il fallait y aller et saisir son va-tout. Il fallait absolument que je sorte dans la rue, c'était mon but, et pour sortir, il fallait inventer ce que j'ai inventé.

$H$ : À un moment tu reconnais les voix, pas de ceux qui donnaient les ordres, mais des subalternes. Ils semblaient te connaître et toi aussi.

$\mathrm{S}$ : Ce qui s'est passé, c'est que ces gens qui m'ont frappé, je les connaissais tous. Je les connaissais, ils ne me connaissaient pas mais ils m'avaient vu pour une raison très simple. Ils faisaient partie de l'équipe qui s'occupait du Parti socialiste. Ils ont fait irruption dans l'appartement de mes parents en août 1974 et ils sont restés pendant cinq jours. J'étais rentré chez mes parents et je suis sorti de justesse. Ces gens-là ont surveillé la maison de mes parents pendant un an. Et le problème, c'est que mon épouse habitait le bâtiment en face mais que eux ne le savaient pas. Et moi, quand j'allais voir mon épouse, je voyais les gens qui surveillaient l'appartement. J'habitais dans le quartier depuis quinze ans, donc je savais bien qui était du quartier et qui était étranger. Je le savais car trois ou quatre fois, ils ont surveillé l'appartement de mes parents depuis l'étage de mon épouse. C'était des escaliers ouverts, ils m'avaient vu mais ils ne m'identifiaient pas avec la photo qu'ils avaient de moi. Quand ils m'ont arrêté, ils m'ont dit, « mais on te connaît ! , j'ai dit, « oui, oui », pour qu'ils ne fassent pas la relation avec mon épouse. "À l'école d'économie, on s'est croisés ». Ils étaient allés là pour m'attraper et ils ont dit, « oui, on s'est croisés à l'école d'économie, on vous a vu là-bas. » Donc je les connaissais de vue, pas plus.

Dans cette équipe-là, il y avait des gens qui ont marqué l'histoire chilienne par le mauvais côté. Par exemple il y avait Osvaldo Romo, un tortionnaire connu qui est mort en prison, un type qui a été dénoncé par de nombreux témoins, un homme très sauvage qui a assassiné des gens. Dans le groupe qui avait investi l'appartement de mes parents et qui m'a torturé, il y avait le colonel Barriga. C'est un homme qui a tué des gens avec un couteau ; et donc, ce n'était pas des tendres. Il s'est suicidé il y a quelques années. Il s'est lancé du $18^{\mathrm{e}}$ étage. On avait pratiqué una funa. Quand on avait détecté quelqu'un qui avait torturé, on allait devant chez lui et on faisait du bruit. Et on filait des tracts à tous les voisins pour qu'ils sachent quel type d'individu habitait là. Et pour lui, on avait fait una funa. Donc, il n'a pas supporté et en plus il savait qu'il allait être appelé à témoigner et être emprisonné. Il s'est donc jeté du $18^{\mathrm{e}}$ étage. C'est Pedro Matta qui est un camarade qui m'a prévenu à Paris. Il m'a envoyé un e-mail et il m'a dit, « qu'est-ce que tu penses de cette nouvelle? » et j'ai répondu, « dommage qu'il ne se soit pas jeté du $19^{\mathrm{e}} \gg$ (rires). 
$H$ : Tu as écrit ce livre et, ayant réussi à échapper à tes tortionnaires, tu as été le seul en mesure de témoigner directement sur la torture sous Pinochet.

$\mathrm{S}$ : Oui, c'est pour cela qu'on m'a invité au procès. C'était important pour les faits historiques. Ce qui m'a surpris, c'est l'accueil du juge qui a été vraiment très chaleureux, des greffiers qui sont venus me saluer qui connaissaient mon cas. C'était de petits détails comme cela qui sont assez impressionnants. Il y avait la police aussi qui voulait me voir. Je l'ai appris par la suite. Je ne les ai pas vus. Je ne sais pas pourquoi. Il y avait un service d'investigation de police civile qui a créé un département s'occupant des droits de l'homme. Donc les choses avaient quand même changé au Chili.

$H$ : Le fait que les tortures soient dénoncées officiellement, cela a-t-il changé quelque chose pour toi personnellement?

$\mathrm{S}$ : Oui et non, car quelques années auparavant on m'avait invité à faire un témoignage contre Pinochet. Je me souviens très bien. C'était un jour où j'étais assez débordé chez moi, je m'occupais de mes enfants. C'est une femme qui m'a appelé. Elle m'a dit, « c'est vous, Sergio Zamora? Je voulais vous voir, il faut que vous témoigniez. " J'ai répondu, " écoutez, arrêtons cela, parce que j'ai déjà écrit un bouquin et pour moi, c'est fait. » Maintenant, je me rends compte qu'elle avait raison, pas moi. Je vous raconte cela, parce que quand j'ai écrit ce livre, il y avait très peu de témoignages.

Quand j'ai écrit le livre en 1993, je me souviens que les gens de gauche chiliens en général disaient, "pourquoi tu fais cela quand il faut oublier, il ne faut pas remettre cela sur le tapis ». Mais je me souviens que tout cela a changé après que Pinochet s'est fait arrêter à Londres, mais avant, ce n'était pas évident.

Du fait que j'ai écrit ce livre et le deuxième Après septembre qui complète celui-là, j'avais la conscience tranquille en tant que témoin de l'histoire. J'avais accompli mon rôle, c'était fait. Alors le fait d'aller au Chili en 2006 et de participer à un tel procès, c'était important pour moi. Mais j'aurais bien voulu qu'il ait lieu vingt ou trente ans avant et pas si tard. Mais c'était tout de même important.

$\mathrm{H}$ : Oui, important pour d'autres gens et pour la jeune génération.

$\mathrm{S}$ : C'était important pour les gens qui m'ont invité et qui y ont participé. Je ne dis pas que cela n'était pas important pour moi. Mais le fait que j'avais déjà écrit le livre, que j'avais déjà témoigné, la charge émotionnelle était beaucoup moins forte que si cela n'avait pas été le cas.

$H$ : Dans ce que tu écris maintenant, dans tes nouvelles en particulier, est-ce qu'il n'y a pas une difficulté à vraiment se sentir bien ici et ailleurs, entre France et Chili ?

$S$ : Non. Quand je suis arrivé en France, les trois ou quatre premières années, mon épouse et moi, la seule chose que nous voulions, c'était rentrer au Chili. Mais comme on ne le pouvait pas - on le savait - le Chili nous manquait énormément. C'était tous les jours. On se rappelait, «Ah, ce fruit n'est pas aussi bon qu'au Chili... ». Par la suite le temps a passé. Maintenant, cela va faire presque trente ans que nous vivons en France. On a vécu plus longtemps en France qu'au Chili. Maintenant le rapport qu'on a avec le Chili n'est plus le même qu'il y a vingt-cinq ans. J'en parlais avec des amis. Si on m'invite à vivre au Chili, je dirai non. Il y a mes enfants, mon petit-fils, donc tout cela compte. On est des déracinés qui ont pris racine. Pour tous ceux qui comme moi - et ils sont nombreux - ont été forcé de vivre loin de leur pays, ce n'est pas une vie normale parce que, quelque part, on a perdu quelque chose. Mais bon, c'est comme cela, la vie continue, on s'habitue. 
$\mathrm{H}$ : Dans les années terribles d'angoisse permanente, tout à l'heure tu as parlé du livre, L'Orchestre rouge. Y a-t-il d'autres textes, des poèmes qui ont été un point d'appui pour toi ? J'ai lu dans ta première nouvelle du recueil, Un train pour Valparaiso, que tu cites Cervantès, Borges...

S : Disons que oui. Le coup d'Etat, s'il a favorisé quelque chose, c'est la lecture. On passait de longs moments à lire, un peu de tout, des documents politiques, de la littérature... Evidemment on essayait de joindre l'utile à l'agréable, de lire des choses qui pouvaient nous convenir. Je me souviens que j'avais lu des textes qui parlaient plus ou moins d'expériences semblables à celle du Chili qui était quand même une nouveauté pour nous. Mais le 15 mai 1975, ce jour-là, il y a deux textes qui m'ont frappé, L'Orchestre rouge - j'y pensais tout le temps - et aussi le livre d'Hemingway, Pour qui sonne le glas. Quand je suis arrivé au centre de torture, j'ai entendu les cloches. Là, j'ai eu peur en entendant les cloches, parce que le personnage meurt à la fin. Ce n'était pas une inspiration, c'était un rappel.

\section{AUTEURS}

\section{SERGIO ZAMORA}

Sergio Zamora est un écrivain chilien qui vit en France depuis le 15 juin 1975. 